\title{
Behavioural phenotype of Cornelia de Lange syndrome
}

\author{
T P Berney, M Ireland, J Burn
}

\begin{abstract}
A postal questionnaire was used to study 49 individuals with Cornelia de Lange syndrome (including both the classical and the mild forms) to ascertain behavioural phenotype. Ages ranged from early childhood to adulthood (mean age, 10.2 years; SD, 7.8) and the degree of mental retardation from borderline $(10 \%)$, through mild $(8 \%)$, moderate $(18 \%)$, and severe $(20 \%)$ to profound $(43 \%)$. A wide variety of symptoms occurred frequently, notably hyperactivity $(40 \%)$, self injury (44\%), daily aggression (49\%), and sleep disturbance $(55 \%)$. These correlated closely with the presence of an autistic like syndrome and with the degree of mental retardation. The frequency and severity of disturbance, continuing beyond childhood, is important when planning the amount and duration of support required by parents.

(Arch Dis Child 1999;81:333-336)
\end{abstract}

Keywords: Cornelia de Lange syndrome; behaviour; mental retardation; autism

Cornelia de Lange syndrome is a rare malformation syndrome, characterised by mental retardation, short stature, limb abnormalities, and distinctive facial features. It has been reviewed recently ${ }^{1}$ and the clinical dichotomy into "classical" and "mild" cases is now generally accepted. Those in the mild group show less retardation of growth and intellect. They seldom have the more severe limb reduction defects or congenital heart defects, although the characteristic facial features may be the same in both groups. ${ }^{2}$

The syndrome has been associated with certain behavioural and temperamental features, the first descriptions of a characteristic pattern of behaviour being made in 1971 and subsequently supplemented by further case descriptions. ${ }^{3-5}$ These accounts, based on the videotaped records of 13 children, emphasised self injury and the autistic-like qualities that they displayed, providing the clinical material to coin the term "behavioral phenotype" ${ }^{6}$ Since then the low incidence and inconsistent diagnosis have been major obstacles to the better definition of these aspects of the syndrome. A detailed clinical study found a high rate of misdiagnosis. ${ }^{2}$

A postal survey of 138 families found the children to be hypersensitive, dysrhythmic, hyperactive, and impulsive. ${ }^{78}$ Underlying medical conditions were frequent and there was a noticeable sensitivity to the effects of medication. There was no specific association with neuropsychiatric symptoms such as compulsive self injury or aggression. A questionnaire study of 27 children found feeding problems, overactivity, irritability, self injury, and stereotyped behaviours to be characteristic of most of the group who were severely handicapped. ${ }^{9}$

Our study followed on from a clinical study of de Lange syndrome with the aim of further delineating the behavioural phenotype, highlighting any differences between the classical and mild groups.

\section{Methods}

The individuals in this study had previously been ascertained through canvassing paediatrics, clinical genetics, and child psychiatry in the UK and the Cornelia de Lange Syndrome Foundation. The diagnosis was confirmed by examination by one of the authors (MI), who classified the subject as mild or classical predominantly on facial appearance reinforced by data on the presence or absence of severe limb defects, overall growth, and the degree of mental retardation. The carers were then sent a postal questionnaire in two instalments.

Most of the first instalment was dedicated to the development and behavioural characteristics of the individual, being derived from (and including) the questionnaire developed by the Society for the Scientific Study of Behavioural Phenotypes. ${ }^{10}$ It covered those areas identified in the literature as associated with the de Lange syndrome, including self injury, impaired social relationships, and repetitive or unusual motor behaviour. There was a particular emphasis on symptoms associated with autism, asking about both the current state and, specifically, about symptomatology at the age of 3-5 years. The questionnaire was piloted on six of the subjects, scattered across the whole age range, whose parents were subsequently interviewed, and any questions that gave a discrepant response were modified or discarded.

Also included in the first instalment was the "EAS temperament questionnaire" to be completed on behalf of the subject as well as by each of the parents.

In the second instalment were the "dimensions of temperament questionnaire" (revised) (DOTS-R $)^{12}$ - again a copy being provided for the subject and for each parent - as well as a modified "autism behavioural checklist". ${ }^{13}$ The latter was used to identify symptoms, rather than to give a diagnostic score, and used a four point Likert scale instead of the original yes/no coding (which had been the subject of earlier criticism). ${ }^{14}$ 
Table 1 Symptom frequency

\begin{tabular}{llll}
\hline Symptom & Mild MR $(n=9)$ & Severe MR $(n=40)$ & Total $(n=49)$ \\
\hline $\begin{array}{l}\text { Sleep problems } \\
\quad \text { At least one symptom }\end{array}$ & $3(33)$ & $24(60)$ & $27(55)$ \\
$\quad \begin{array}{l}\text { Three or more symptoms } \\
\text { Current GI symptoms }\end{array}$ & 0 & $12(30)$ & $12(24)$ \\
$\quad$ At least one symptom & $4(44)$ & $29(73)$ & $33(67)$ \\
$\quad$ All three symptoms & 0 & $4(10)$ & $4(8)$ \\
$\begin{array}{l}\text { Hyperactivity } \\
\quad \text { Any one symptom }\end{array}$ & $3(33)$ & $33(83)^{\star \star}$ & $36(74)$ \\
$\quad$ All four symptoms & 0 & $7(18)$ & $7(14)$ \\
$\quad \begin{array}{l}\text { Self injury } \\
\quad \text { Occurring at least occasionally }\end{array}$ & $2(22)$ & $25(64)^{\star}$ & $27(56)$ \\
$\quad$ Severe & $1(11)$ & $10(26)$ & $11(23)$ \\
Aggression & & & $35(75)$ \\
$\quad$ Occurring at least occasionally & $6(67)$ & $29(76)$ & $23(49)$ \\
$\quad$ Occurring daily & $3(33)$ & $20(53)$ & \\
\hline
\end{tabular}

Figures in parenthesis are percentages of those with mild or severe mental retardation (MR).

${ }^{\star} \mathrm{p}<0.05 ;{ }^{\star \star} \mathrm{p}<0.01$

GI, gastrointestinal.
The data were analysed using $\chi^{2}$ and hierarchical log linear tests from the Statistical Package for the Social Sciences.

\section{Results}

Of 49 subjects whose families returned the questionnaires, 21 were male and 28 were female. All lived with their own families except for one man who had been resettled from hospital to a community house: however, his carers had known him from early childhood. The age range included childhood $(65 \%)$, adolescence $(20 \%)$, and adulthood (14\%) using 12 years and 19 years as arbitrary cut offs. The degree of mental retardation ranged from borderline $(10 \%)$, through mild (8\%), to moderate $(18 \%)$, severe $(20 \%)$, and profound $(43 \%)$. For this analysis we dichotomised mental retardation into mild (including borderline and mild) and severe (including moderate and profound). Ten cases $(20 \%)$ had severe limb reduction defects, ranging from missing fingers to absent forearms.

Within the classification into classical and mild phenotypes, there was a close association between the somatic features (particularly the dysmorphic facial features and height) and the degree of mental retardation. Female subjects were more severely afflicted, $75 \%$ having the classical phenotype in comparison with only $45 \%$ of the male subjects $(\mathrm{p}<0.05)$.

We found that $26(53 \%)$ patients had the combination of impaired sociability, communication deficits, and restricted repetitive behaviour to a degree that might indicate an autistic disorder, as defined in International Classification of Diseases 10th revision (ICD-10). On the basis of the questionnaire responses, a clinician with particular experience in this field (TPB) made a judgment as to the presence and severity of childhood autism. The results indicated that this was particularly pronounced in $18(37 \%)$, indeterminate in eight $(16 \%)$, and absent in 23 (47\%). There was no significant gender bias. The presence of pronounced autism related closely to the degree of mental retardation, occurring in $0 \%, 25 \%, 22 \%, 40 \%$, and $52 \%$ of those with borderline, mild, moderate, severe, and profound degrees of mental retardation, respectively. When allowance had been made for the degree of mental retardation there was no significant association with the somatic phenotype (the degree of growth retardation and the presence of severe limb deficiencies).

Fourteen of the patients had had seizures at some time (including febrile seizures). Of these one continued to have seizures whereas eight, distributed across the whole age range, were effectively controlled on antiepileptics.

Sleep disturbance (table 1) was present in $55 \%$ of the group and was associated with autism, even after controlling for the somatic phenotype and the degree of mental retardation $\left(p=0.02 ; \chi^{2}=5.2\right)$. The symptoms, occurring at least twice each week, included an irregular sleep pattern, initial or terminal insomnia, interrupted sleep, and excessive daytime drowsiness, $25 \%$ having at least three of these present simultaneously.

Gastrointestinal symptoms (table 1), including dysphagia, indigestion, and constipation, were present in $67 \%$ of the group, all three being present in $8 \%$. The classical phenotype was associated with difficulty in swallowing even after allowance had been made for the degree of mental retardation $(\mathrm{p}=0.01$; $\left.\chi^{2}=6.4\right)$.

Symptoms associated with hyperkinetic disorder were present in $74 \%$ of the patients (table 1 ), including an attention span of less than 10 minutes ( $47 \%$ ), fidgetiness ( $49 \%)$, chaos creating activity (31\%), and overactivity (40\%). Only $14 \%$ had all four symptoms and all were profoundly mentally retarded. Although overall symptomatology was associated strongly with mental retardation $\left(p=0.003 ; \chi^{2}=9.1\right)$ there were two specific exceptions. The symptom of a short attention span was associated with the classical phenotype even after controlling for autism and the degree of mental retardation $\left(p=0.015 ; \chi^{2}=6.1\right)$. Second, the creation of aimless chaos was associated with autism after controlling for the degree of mental retardation and the somatic phenotype $(p=0.003$; $\left.\chi^{2}=8.7\right)$.

Twenty seven $(55 \%)$ of the group showed self injurious behaviour (table 1). Although in most individuals this only caused transient marking, it was more substantial in 11 of the subjects. It took various forms, only $30 \%$ restricting themselves to one site, and consisted predominantly of finger biting, head banging or scratching and, less commonly, wrist biting and eye poking. The number of sites did not relate to any particular characteristic and, in particular, neither to the level of disability nor to the presence of autism.

Self injury was related to the subject's age, being present in $82 \%$ of those over 12 years against $42 \%$ of those under 12 years, a relation that was maintained even after autism, mental retardation, and pain awareness were controlled for $\left(p=0.005 ; \chi^{2}=7.8\right)$. An altered awareness of pain did not relate closely to the occurrence of self injury but was associated with the presence of autism even after controlling for mental retardation and age $(\mathrm{p}=0.03$; $\left.\chi^{2}=5.1\right)$.

In $76 \%$ of cases, self injury was a response to recognisable triggers, which included thwarting or frustration (34\%), anxiety or fear (18\%), 
boredom (13\%), and demand avoidance (5\%). Effective responses were only lasting in nine cases, with most carers finding that most techniques had worked at some time. These included talking to the person (effective for $66 \%$ of those who had tried it), holding him/her $(61 \%)$, removing him/her from the room $(55 \%)$, mechanical restraint $(40 \%)$, and ignoring him/her $(38 \%)$. Few had tried medication and, of those who had, only $33 \%$ had found it useful.

Symptoms of aggression occurred daily in $49 \%$ of this group. Although unrelated to age, sex, or ability, they were associated with autism $\left(p=0.002 ; \chi^{2}=9.7\right)$, a relation that remained even after controlling for ability and age. It was vented on objects $(33 \%)$ and, to a lesser extent, on people $(10 \%)$. Vocal aggression, whether expressed by noises or words, occurred in $25 \%$ of subjects.

There was some overlap between self injury and aggression: aggressive episodes occurred in $92 \%$ of those who caused self injury, compared with $52 \%$ of the remainder $(\mathrm{p}=0.002$; $\left.\chi^{2}=9.7\right)$.

Serious temper tantrums occurred daily in five subjects and weekly in seven-a total of $25 \%$ of the subjects - and a further five had them infrequently. There was no obvious association with any aspect of disability and they did not improve with age.

A cyclical mood disturbance was present in $13(27 \%)$ of the subjects. Here, we sought a change of mood and behaviour lasting for weeks or months that included changes in a number of symptoms, such as appetite and weight, sleep, activity level, irritability, interest in life, self injury, aggression, sociability, and sexual interest. All 13 had changes in at least three of these symptoms and 11 had changes in at least five. Cyclical change was related to age, $77 \%$ being over 12 years, compared with only $20 \%$ of those who did not show such changes $\left(\mathrm{p}=0.001 ; \chi^{2}=10.7\right)$. Autism was present in $69 \%$ of those with cyclical change and in only $26 \%$ of the remainder $\left(p=0.03 ; \chi^{2}=5.0\right)$, associations that remained significant even after controlling for the degree of mental retardation

\section{Discussion}

Overall, the group was severely handicapped with a combination of mental retardation, physical disability, and autism. There was a high prevalence of distressing behavioural morbidity and it is noteworthy that this did not diminish with age; indeed, some symptoms, such as self injury and a cyclical shift in mood,

Table 2 Behavioural disturbance and autism

\begin{tabular}{lll}
\hline Symptom & Autistic $(n=30)$ & Not autistic $(n=11)$ \\
\hline Any symptom of sleep disturbance & $14(78)$ & $9(39)^{\star}$ \\
Current gastrointestinal symptomatology & $14(78)$ & $11(48)$ \\
Any symptom of hyperactivity & $16(89)$ & $14(61)^{\star}$ \\
Self injury occurring at least occasionally & $15(83)$ & $7(32)^{\star \star}$ \\
Severe self injury & $7(39)$ & $2(9)^{\star}$ \\
Aggression occurring at least occasionally & $16(89)$ & $15(71)$ \\
Aggression occurring daily & $14(78)$ & $8(38)^{\star}$
\end{tabular}

Figures in parenthesis are percentages of those who clearly have or do not have autistic symptomatology.

${ }^{\star} \mathrm{p}<0.05 ;{ }^{\star \star} \mathrm{p}<0.01$.

\section{Key messages}

- Behaviour disturbance is common in Cornelia de Lange syndrome and becomes more frequent as mental retardation becomes more severe

- Typical features include hyperactivity, self injury, aggressive behaviour, and sleep disturbance

- Self injury was more frequent in those over 12 years age

- An autistic syndrome was a frequent finding, particularly in those with severe mental retardation

appeared to be more frequent in the older group. Some of these shifts may well have represented depressive episodes, unrecognised because of the masking effect of disability, ${ }^{15}$ but the questionnaire was not designed to explore this aspect.

Our study is unusual in that it attempts to identify and take into account the syndrome of autism, although this requires some qualification. The diagnosis is difficult where there is severe mental retardation, which will obscure the more characteristic, positive elements. This makes it difficult enough to identify autism in a live interview, using the appropriate instruments such as the "autism diagnostic instrument" and the "autistic diagnostic observation schedule". ${ }^{16}$ Diagnosis is even more contentious when made by questionnaire which, at its best, should be a screening instrument, identifying cases for a more intensive, live evaluation. Nevertheless, these children had a form of disturbance that was acutely distressing to their families, qualitatively different to the deficits that might be expected simply from the same degree of mental retardation and which, insofar as we could discern, was autistic-like. The results are consistent with other accounts of the behavioural phenotype. ${ }^{17} 18$ They contrast with those of a postal survey of 138 cases in the USA, which emphasised the affectionate, sensitive, and temperamental qualities of the phenotype, although self injury and aggression were identified as an unusually intense response to stress. ${ }^{78}$

The prevalence of autism is related to the degree of mental retardation. ${ }^{19}{ }^{20}$ When allowance had been made for this, there did not seem to be any additional, specific association between the autistic symptomatology and de Lange syndrome. The association of autism with a variety of adverse behavioural disturbance is striking (table 2).

It is an important theoretical point whether individuals with autism or severe mental retardation should be included in the pool of people from which a behavioural phenotype is derived. One view is that they simply represent the extreme end of a continuum and that their characteristics can be discerned, albeit more faintly, in a high proportion of the group. Alternatively, they can be considered to be a distinct category, which has such intense 
symptomatology as to overshadow the subtler and very different phenotype of the nonautistic group. Certainly, such pooling of cases might account for the combination of social and language impairment ascribed to many of the more able cases. ${ }^{1721}$ Our study includes seven subjects who had, at the most, mild mental retardation and who had no evidence of autism. Other than it was unusual for them to be emotional or to cry easily, we could discern no obvious temperamental characteristics.

A diminished responsiveness to pain has been noted to be associated both with mental retardation ${ }^{22}$ and with autism, ${ }^{23}$ and it might contribute to self injury (although this did not emerge in our study). The person's awareness of pain cannot be gauged from their indifference to self injury: rather, it has to be judged by their response to accidental hurt. ${ }^{24}$ We found a clear relation between the presence of a diminished response to pain and autism, which was independent of the effect of mental retardation.

Self injury has been prominent in past descriptions $^{925}$ and our study confirms the association with the older age group. It is linked, however, to the presence of an autistic syndrome rather than simply to more severe mental retardation.

Although symptoms associated with hyperactivity were frequent, the full blown combination of greatly impaired attention and overactivity was present in only 12 subjects. All but one had severe mental retardation, and that patient had an autistic syndrome. Only one, an adolescent boy, would fall into the category of hyperkinetic disorder (F90, ICD-10). Of the others, seven had autism and three would meet the criteria for "overactive disorder associated with mental retardation and stereotyped movements" (F84.4, ICD-10).

Periodicity pervades normal development and an intermittent disturbance does not automatically indicate an affective disorder. Nevertheless, it is an important symptom and one that merits closer inquiry, particularly as cyclical mood change can be associated with adolescence and autism, ${ }^{26}$ and can amplify or mimic the latter. ${ }^{27}$

Analysis of the temperament questionnaires did not show any pattern of traits. However, this is unsurprising, given that there were only six cases who had neither severe mental retardation nor autistic symptomatology, both elements that might be expected to obscure the picture.

Overall, among those with confirmed de Lange syndrome, there is a very high prevalence of wide ranging behavioural disturbance. This must be taken into account in the provision of services for these families.
M Ireland is an MRC clinician scientist fellow.

1 Ireland M. Cornelia de Lange syndrome: clinical features, common complications and long-term prognosis. Curr Paediatr 1996;6:69-73.

2 Ireland M, Donnai D, Burn J. Brachmann-de Lange syndrome. Delineation of the clinical phenotype. Am f Med Genet 1993;47:959-64.

3 Bryson Y, Sakati N, Nyhan WL, Fish CH. Self-mutilative behavior in the Cornelia de Lange syndrome. Am $\mathcal{F}$ Ment Defic 1971;76:319-24

4 Johnson HG, Ekman P, Friesen W, Nyhan WL, Shear C. A behavioral phenotype in the de Lange syndrome. Pediatr Res 1976;10:843-50.

5 Shear CS, Nyhan WL, Kirman BH, Stern J. Self-mutilative behavior as a feature of the de Lange syndrome. $\mathcal{F}$ Pediatr 1971;78:506-9

6 Nyhan WL. Behavioral phenotypes in organic genetic disease. Presidential address to the Society for Pediatric Research, May 1, 1971. Pediatr Res 1972;6:1-9.

7 Gualtieri CT. Behaviour in the Cornelia de Lange syndrome. In: Neuropsychiatry and behavioral pharmacology. New York: Springer-Verlag, 1991:173-86.

8 Gualtieri CT. Behavior in the Cornelia de Lange syndrome. Reaching out: the newsletter of the Cornelia de Lange Syndrome Foundation 1993;12(5).

9 Sarinski K. Communication, social-emotional developmental and parenting stress in Cornelia-de-Lange syndrome. $\mathcal{F}$ Intellect Disabil Res 1997;41:70-5.

10 O'Brien G. Behavioural phenotypes and their measurement. Dev Med Child Neurol 1992;34:365-7.

11 Buss AH, Plomin R. Theory and measurement of EAS. In: Temperament: early developing personality traits. Hillsdale, New Jersy: Lawrence Erlbaum Associates, 1984:84-104.

12 Windle M, Lerner RM. Reassessing the dimensions of temperamental individuality across the life span: the revised dimensions of temperament survey (DOTS-R). Fournal of Adolescent Research 1986;1:213-30.

13 Krug DA, Arick JR, Almond PJ. The autism behavior checklist. Portland, Oregon: ASIEP Education Company, 1988

14 du-Verglas G, Banks SR, Guyer KE. Clinical effects of fenfluramine on children with autism: a review of the research. f Autism Dev Disord 1988;18:297-308.

15 Marston GM, Perry DW, Roy A. Manifestation of depression in people with intellectual disability. F Intellect Disabil Res 1997;41:476-80.

16 le Couteur A, Rutter M, Lord C, et al. Autism diagnostic interview: a standardized investigator-based instrument. $\mathcal{f}$ Autism Dev Disord 1989;19:363-87.

17 Goodban MT. Survey of speech and language skills with prognostic indicators in 116 patients with Cornelia de Lange syndrome. Am f Med Genet 1993;47:1059-63.

18 Jackson L, Kline AD, Barr MA, Koch S. de Lange syndrome: a clinical review of 310 individuals. Am $\mathcal{F}$ Med Genet 1993;47:940-6.

19 Ehlers S, Gillberg C. The epidemiology of Asperger syndrome: a total population study. F Child Psychol Psychiatry 1993;34:1327-50.

20 Wing L, Gould J. Severe impairments of social interaction and associated abnormalities in children: epidemiology and classification. 7 Autism Dev Disord 1979;9:11-29.

21 Moeschler JB, Graham J, Jr. Mild Brachmann-de Lange syndrome. Phenotypic and developmental characteristics of mildly affected individuals. Am F Med Genet 1993;47: 969-76.

22 Biersdorff KK. Incidence of significantly altered pain experience among individuals with developmental disabilities. Am ₹ Ment Retard 1994;98:619-31.

23 Gillberg C, Terenius L, Lonnerholm G. Endorphin activity in childhood psychosis: spinal fluid levels in 24 cases. Arch Gen Psychiatry 1985;42:780-3.

24 Stengel E, Oldham AJ, Ehrenberg ASD. Reactions of low-grade mental defectives to pain. Fournal of Mental Science 1958;104:434-8.

25 Rohahn J. Epidemiology and topographic taxonomy of selfinjurious behaviour. In: Thompson T, Gray DB, eds. Destructive behaviour in developmental disabilities. London: Sage, 1994:49-67.

26 Berney TP, Jones PM. Manic depressive disorder in mental handicap. Australia and New Zealand fournal of Developmental Disability 1988;14:219-25.

27 Kerbeshian J, Burd L, Fisher W. Lithium carbonate in the treatment of two patients with infantile autism and atypical bipolar symptomatology. F Clin Psychopharmacol 1987;7: $401-5$. 